\title{
Accuracy assessment and position correction for low-cost non-differential GPS as applied on an industrial peat bog
}

\author{
N.M. Holden ${ }^{\mathrm{a}, *}$, A. Comparetti ${ }^{\mathrm{b}}$, S.M. Ward ${ }^{\mathrm{a}}$, \\ E.A. McGovern a,c \\ a Department of Agricultural and Food Engineering, University College Dublin, Earlsfort Terrace, \\ Dublin 2, Ireland \\ b Dipartimento di Ingegneria e Tecnologie Agro-Forestali (Sezione Meccanica), \\ Universitá degli Studi di Palermo, 13, Viale delle Scienze, Palermo, Italy \\ ${ }^{\mathrm{c}}$ Department of Geomatics, Dublin Institute of Technology, Bolton Street, Dublin 1, Ireland
}

Received 23 February 1999; received in revised form 24 May 1999; accepted 31 May 1999

\begin{abstract}
A low-cost, non-differentially corrected hand-held GPS receiver was tested on an industrial peat production bog. A correction procedure ('pseudo-differential correction') was derived that corrected data points to the nearest position on a line defining the centre of each $15-\mathrm{m}$ wide field. The result was a corrected log of track points for each field for all points lying along the field. It was found that the mean orthogonal distance from a field centreline was linearly correlated with mean uncorrected GPS data error $\left(r^{2}=0.99\right)$ such that as GPS error increased so the accuracy obtained by correction decreased. For a signal with a mean uncorrected error of $\sim 30 \mathrm{~m}$ it was possible to reduce the error to $\sim 12 \mathrm{~m}$. The results are discussed within the design requirements of a precision peat production system for peat energy. It is concluded that low-cost GPS could be used without differential correction as part of a precision peat production system because over $80 \%$ of the time positional error could be constrained to within $15 \mathrm{~m}$. When compared with the perceived patterns of variability and the $30-\mathrm{m}$ resolution of Landsat imagery which can be used for making application maps, this is acceptable. (C) 1999 Elsevier Science B.V. All rights reserved.
\end{abstract}

Keywords: Positioning accuracy; GPS; Differential correction; Milled peat

* Corresponding author. Tel.: + 353-1-7067460; fax: + 353-1-7067481.

E-mail address: peat@ucd.ie (N.M. Holden)

0168-1699/99/\$ - see front matter (C) 1999 Elsevier Science B.V. All rights reserved.

PII: S0168-1699(99)00031-9 


\section{Introduction}

Peat is one of Ireland's indigenous sources of energy. Industrial utilisation began (by Bord na Móna, The Irish Peat Board) in the 1930s. A production bog is prepared by installing a main drain so that the bog will dry and shrink and the load bearing capacity will increase until it can support a machine. After this, parallel field drains are installed to a depth of $1 \mathrm{~m}$, with a spacing of $15 \mathrm{~m}$ to decrease the surface water content further. Once complete, the bog is laid out as a series of parallel $15-\mathrm{m}$ wide fields, up to $2 \mathrm{~km}$ long, with 'headlands' at each end to facilitate access. The peat is 'harvested' in a cycle that is governed by atmospheric drying. The surface of the bog is milled (scarified into particulate peat called 'milled peat') to a depth of $\sim 50 \mathrm{~mm}$ and allowed to dry for up to $96 \mathrm{~h}$. This is performed by a tractor towed miller moving at $\sim 20 \mathrm{~km} \mathrm{~h}^{-1}$, which has three powered rotating drums with teeth that rip the peat surface in a swath 7.5-m wide. It takes two passes to mill one field, and turns are on the headlands. During drying, harrowing is used to increase the rate of water loss, with a final target water content of $45 \%$ required. Once dry, the milled peat is lifted into stockpiles for storage and later moved by train to power stations for electricity generation. There are two stockpiling strategies commonly used in Ireland. The PECO system is used for $85 \%$ of production: the milled peat is collected into piles about $12 \mathrm{~m}$ wide, $4 \mathrm{~m}$ high and up to $2 \mathrm{~km}$ long. The pile sits on a field surrounded by ten source fields. The alternative system (HAKU) collects the peat in large central stockpiles $20-30 \mathrm{~m}$ wide, 50-100 $\mathrm{m}$ long and up to $10 \mathrm{~m}$ high. Even though $10 \%$ of the bog is used for storage with the PECO system, it is preferred because production costs are lower.

Currently the management of the drying stage of harvesting is based on weather forecasts to predict when a suitable 4-day drying period will occur. Once a window of opportunity is predicted an area of bog will be milled. The milling depth will be adjusted to between 30 and $50 \mathrm{~mm}$ by the driver, using his judgement, to try and ensure a product of uniform water content. The efficiency of this is dependent on the driver's interest and fatigue. Work is currently being undertaken to develop an automated adjustment system that accounts for miller sinkage which could be adapted for automated variable depth milling. The peat is most valuable when close to target water content ( $45 \%$ wet weight basis) with little variability. There are three main factors which influence drying on the bog: (i) peat type and density (LaComber et al., 1997), (ii) the weather, and (iii) the milling depth (von Ow et al., 1996). Only the milling depth can be influenced during production, and it needs to be adjusted in relation to peat density and drying potential. The density can be mapped from satellite imagery (McGovern et al., 1998). The mapping is based on Landsat Thematic Mapper imagery which has good coverage in the infra-red wavelengths, but a poor resolution $(30 \mathrm{~m})$. Imagery with a better resolution is available, but cannot produce sufficiently accurate density prediction. Given that it is possible to produce sufficiently accurate (but spatially imprecise) density maps, it should be possible to implement a 
management system for peat production that adjusts milling depth based on density variation. Field evidence available suggests that density varies over distances of tens to hundreds of metres, thus the resolution of the imagery should be sufficient unless there are significant height differences between adjacent fields. Work is currently being undertaken with historical images to evaluate the rate of change of density with depth so that it will be possible to establish the useable life-span of an image once obtained. It should also be possible to adjust depth based on predicted drying conditions, thus allowing production during shorter windows of opportunity which could be important in years when the weather is very poor. The application of precision agriculture methods to industrial peat production should permit (i) improved efficiency, (ii) better returns in marginal years when the weather is poor, and (iii) more profit (Ward and Holden, 1998). In order to implement a precision milling strategy a machine must be locatable within the resolution of the imagery (i.e. $30 \mathrm{~m}$ ) and within the resolution of a field $(15 \mathrm{~m})$. At the current time there are limited differential correction services in Ireland. Wide area satellite systems and coastal marine beacon systems are available, but both have limitations with respect to the industry. The cost of implementation of a machine positioning system for a large fleet of machines $(\sim 200)$ is significantly increased if differential correction is used which is, at the present time, off putting to the industry because the product is low-value, high volume. The marine beacon system provides least reliable coverage in the Irish Midlands which is where most industrial peat production occurs.

From a positioning point-of-view, the peat field has the characteristic that once a wheeled machine enters a field, it cannot stray from the area defined by the drains (i.e. a track $15 \mathrm{~m}$ wide), and therefore the field structure provides a basis for developing a position correction system for use with non-differentially corrected GPS. The cost of such a system to the industry would be small compared to implementing a differential system because all the bogs have been carefully surveyed, thus a geo-referenced structure is already in place. This paper addresses the question of whether the structure of the peat bog can be used to implement a 'pseudo-differential correction' on GPS track data by using the parallel field drains $15 \mathrm{~m}$ apart. The knowledge that a machine is restricted to a particular field area can be used to 'correct' GPS position data because it can be assumed that the machine will always travel in a relatively straight line. Beuche and Hellebrand (1998) suggested that, when moving in a straight line, regression of position with time could be used to interpolate position during times when differential correction signal is lost. In this case, the fact that the machine is restricted in its travel can be used to estimate where it should be on the field. The principle of the method relies on the fields on the bog being described in terms of lines. Each $15-\mathrm{m}$ wide field can be described by a single straight line, and the position predicted by the GPS can be evaluated in terms of deviation from the straight line used to describe the field position. 


\section{Theory}

\subsection{Description of the bog field structure}

The centreline of a field on a production bog (Fig. 1) is described by the equation for a straight line:

$$
a x+b y+c=0
$$

the $x$ and $y$ co-ordinates of which are drawn (in metres) from the Irish National Grid, and the fitting parameters $(a, b$, and $c$ ) are calculated using the end-point co-ordinates of the line. The boundary between the fields and the headlands can also be described by straight lines using a similar equation. The distance and direction of a point of known co-ordinates from that line defined by Eq. (1) is then:

$$
d=\frac{a x+b y+c}{\sqrt{a^{2}+b^{2}}}
$$

where $d$ is orthogonal distance $(\mathrm{m})$ from the line. The sign of $d$ indicates which side of the line the point in question lies. It is therefore possible to describe the structure of the production bog using a series of parallel straight lines to represent the centrelines of the fields, and another series of straight lines to define the boundary between the fields and the headlands.

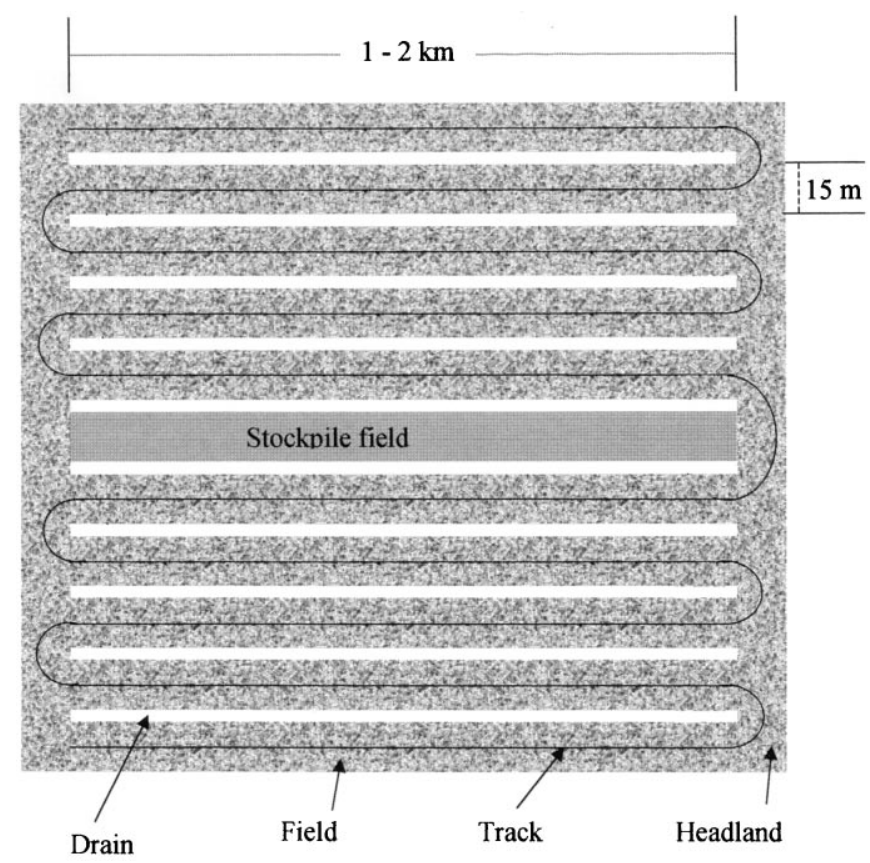

Fig. 1. Schematic representation of fields on a production peat bog, and their description by centrelines. 


\subsection{Position of machine relative to the headland boundary}

The boundaries between the fields and headlands can be defined so that any point on a field has a negative value with respect to both boundary lines. From this it is possible to estimate whether a vehicle is on a field or on a headland, using logical operators. In addition to position relative to the boundary, the change in heading between data points and the distance between consecutive points should also indicate whether the movement of a machine is in a straight line, or describing a curve at slower speed during a turn on the headland.

\subsection{Position on the field}

A milling machine will always be within $3.75 \mathrm{~m}$ of the centreline of the field it is travelling along because the miller is $7.5 \mathrm{~m}$ wide and is constrained to a $15-\mathrm{m}$ wide field. It is therefore possible to find the nearest point on the centreline to a non-differentially corrected position as estimated by GPS. It is assumed that 'correcting' points to the nearest position on the centreline will constrain error to a magnitude less than that of non-differentially corrected data, and to a level useable for industrial application. Using the nearest point on the centreline will constrain lateral error (orthogonal to the direction of travel) to $3.75 \mathrm{~m}$ and will create an unknown error in the direction of travel. The calculation is represented graphically in Fig. 2. The orthogonal distance of a GPS position to the centreline (A in Fig. 2) can be calculated using an absolute form of Eq. (2) to remove the sign. The distance between the known end point of the field (used to calculate the straight line parameters) and the GPS position (B) can be calculated by Pythagorus' theorem since this is the distance between two points in an orthogonal co-ordinate system. From $\mathrm{A}$ and $\mathrm{B}$ the distance $(\mathrm{C})$ along the field centreline equivalent to the GPS position is also calculated using Pythagorus' theorem. The grid bearing of the field line $(\alpha)$ can be calculated, and from this information the adjusted point co-ordinates $\left(E_{\text {corrected }}, N_{\text {corrected }}\right)$ are calculated by converting from Polar to Cartesian co-ordinates using the equation:

$$
E_{\text {corrected }}=E_{\text {known }}+C \cos \alpha \quad \text { and } \quad N_{\text {corrected }}=N_{\text {known }}+C \sin \alpha
$$

where the known co-ordinates $\left(E_{\text {known }}, N_{\text {known }}\right)$ are those used to calculate B.

\subsection{Which field?}

In peat milling operations, it is normal for the machine to always move onto the next available field in sequence, thus if the first field location is known, then all subsequent fields traversed will be known based on the direction of the first turn. If the assumption of always moving onto the next available field is not used, this raises the question of whether a low-cost non-differentially corrected GPS unit can be used to establish which field the machine has turned on to. The nearest field after turning can be found based on the distance from all field centrelines (as calculated using Eq. (2)) to the current GPS position. The minimum distance is used as the 


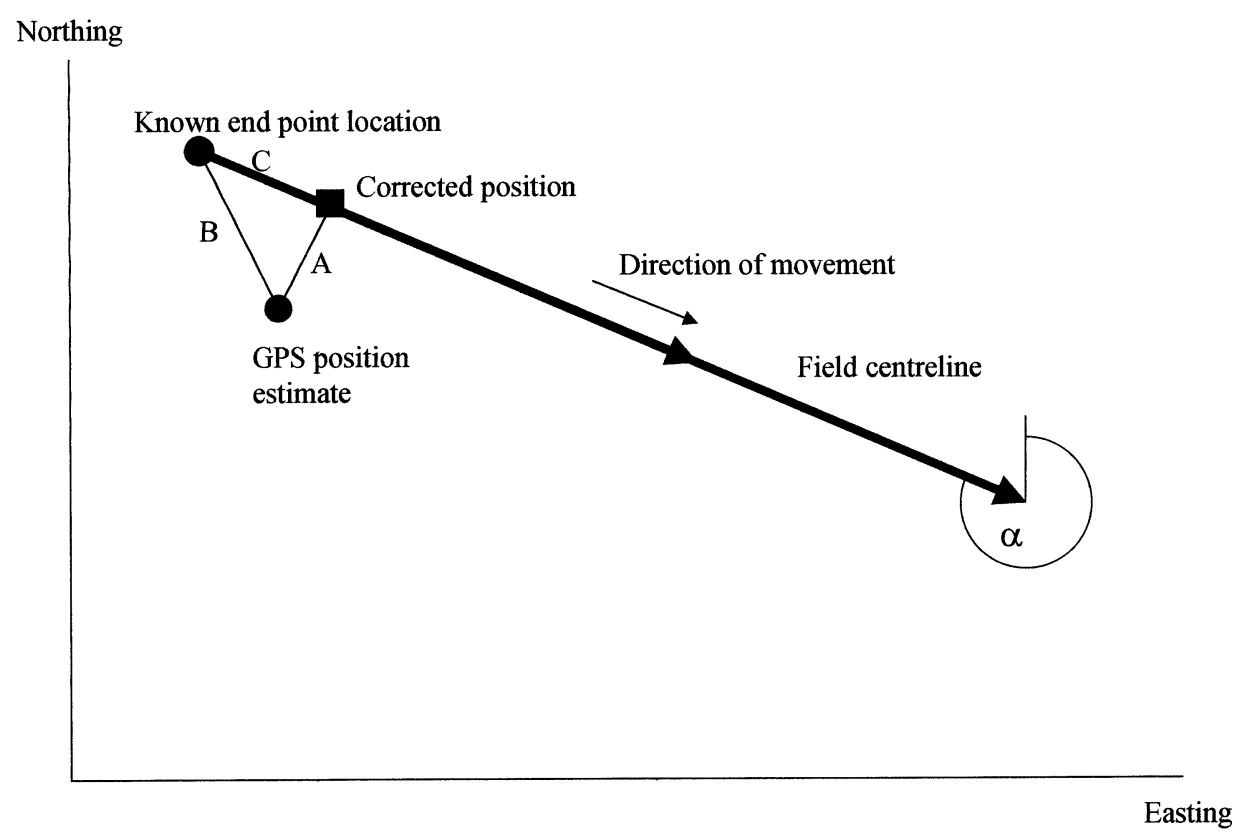

Fig. 2. Graphical representation of the calculation used for GPS position correction using field structure. (A) Orthogonal distance from the centreline to the position predicted by the GPS. (B) The distance between the GPS position and the known end point of the field. (C) The distance along the field centreline equivalent to the GPS position.

basis for determining the field for correction to centreline, hence inaccuracies may occur.

\section{Materials and methods}

The research site was located at Noggusboy bog (Bord na Móna Boora works) in the Irish Midlands, near Tullamore, Co. Offaly $\left(7^{\circ} 51^{\prime} \mathrm{W}, 53^{\circ} 26^{\prime} \mathrm{N}\right)$. The procedure was to survey the bog to produce a base map and then to travel the bog on a tractor equipped with kinematic carrier phase GPS, a marine beacon differential GPS and a non-corrected, cheap hand-held unit. The carrier phase data were used as the 'baseline' data for vehicle position at any particular time.

The base map was constructed by surveying the points in the centre of 15 fields at the boundary between the field and the headland using a Trimble $4000 \mathrm{SSi}$ carrier phase correction GPS system with an accuracy/precision of 5-10 mm (2dRMS). The raw position data were post-processed and converted into Irish National Grid co-ordinates and the bog structure defined using straight lines (Eq. (1)). The resulting map of the test site was annotated to indicate which fields could be travelled and which were being used to store stockpiles (Fig. 3). 
Track data were acquired on 14th September, 1998 using: (i) a low-cost handheld unit, the Garmin GPS 12XL Personal Navigator with an antenna mounted in the centre of the tractor roof (referred to as Garmin), (ii) a Trimble PRO XRS DGPS with marine beacon signal antenna elevated above the tractor roof (referred to as PRO XRS), and (iii) a Trimble 4000 SSi kinematic carrier-phase GPS also with an elevated antenna (referred to as SSi) and a base station located at a site used in a previous survey (McGovern et al., 1998). Additional data had also been obtained previously using just the Garmin unit with the antenna mounted on the roof (30th July, 1998; 'good' data set) and on the wheel arch of the tractor (12th June, 1998; 'poor' data set). The mounting of the antenna on the tractor wheel arch resulted in a signal degradation depending on the direction of travel due to the tractor cab interfering with signal from satellites in part of the sky.

A stopwatch was synchronised to GPS time and the three GPS receivers were set to $\log$ position every $5 \mathrm{~s}$ (PRO XRS and SSi units, allowing 6 hours' data acquisition) and $10 \mathrm{~s}$ (Garmin unit, allowing 2 hours' data acquisition). The tractor was driven along each of the ten available fields in the test area consecutively, and then in the reverse direction (generating data for 20 fields on a sampling occasion). At the start and end point of each field the stopwatch time was noted and subsequently used to determine which data were associated with which field. The track data were processed in a spreadsheet to calculate corrections to position.

A series of questions were evaluated using the Garmin data to determine its potential in the industrial production system: (i) is it possible to tell whether the machine is moving on a field or turning at a headland? (ii) can machine position along a field be estimated with any reliability? (iii) can the actual field the machine

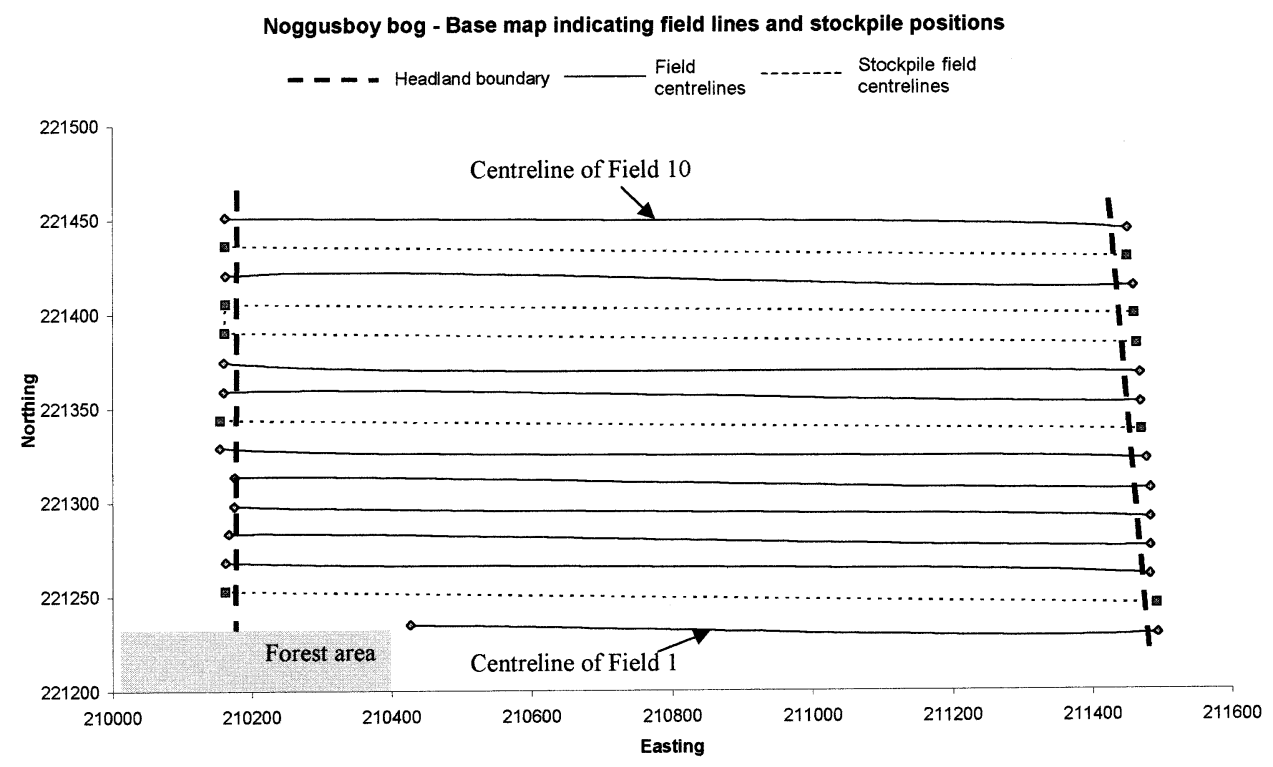

Fig. 3. Surveyed field centrelines and field end boundary lines defining headland positions. 
Table 1

Correction errors (m) and raw data point errors (m) for SSi, PRO XRS and Garmin GPS systems ${ }^{\mathrm{a}}$

\begin{tabular}{llccccc}
\hline System & Data type & Run 1 & Run 2 & Mean & Adjusted error & Improvement \\
\hline SSi & Post processed & 3 & 1 & 2 & & \\
PRO XRS & As recorded & 52 & 27 & 40 & 37 & 19 \\
& Corrected & 29 & 12 & 21 & 18 & 11 \\
Garmin & As recorded & 27 & 18 & 22 & 20 & \\
& Corrected & 14 & 9 & 11 & 9 & \\
& & & & &
\end{tabular}

${ }^{\text {a }}$ All data collected 14th September, 1998.

is on be found with reasonable reliability if it is not assumed that consecutive fields are travelled?

\section{Results}

Applying the correction method to the SSi position data measured on 14th September, 1998, indicated a mean offset of $2 \mathrm{~m}$ for all 20 fields travelled on that day (Table 1). This value indicated the inherent error in the correction method due to driver deviation from the centreline and antenna offset from the centre of the tractor.

It was possible to establish when the machine was on a field or headland with good results using Garmin data. All turns were identified using the boundary line method with a general over-estimation as expected. For most turns only one datum was recorded by the Garmin during time on the headland but the method generally estimated the first two points after the turn as being headland. This error was $\sim 50$ $\mathrm{m}$ and accounted for about $4 \%$ of field length.

The magnitude of the error after applying the correction method was compared with the inherent error by calculating the shortest straight-line distance between the GPS position (or corrected position) and the SSi unit position at that time (Table 1). The mean error was adjusted by subtracting the error due to deviation from the centreline to derive an estimate of accuracy improvement. For the Garmin system the accuracy improvement was about $11 \mathrm{~m}$. It was observed that there was a linear relationship between mean orthogonal offset from the field centreline (A in Fig. 2) and the correction error $\left(e_{\mathrm{c}}\right.$, distance between SSi unit position and corrected position). This could be described by the relationship (based on absolute error values):

$$
e_{\mathrm{c}}=0.6516 A+0.5331 \quad R^{2}=0.9554
$$

From this relationship it was possible to estimate correction error for any GPS dataset aligned to a straight line using the correction method presented (i.e. those obtained for previous tests). The mean orthogonal error for the other Garmin data sets was $11 \mathrm{~m}$ (good data set) and $17 \mathrm{~m}$ (poor data set), which resulted in the mean absolute error estimate for those data sets by correction to be 8 and $11 \mathrm{~m}$. The 
performance of the Garmin unit was consistent (even under adverse conditions) over a period of 6 months. The effect of correction to the field centreline can be clearly seen (Fig. 4).

The PRO XRS unit with marine beacon correction did not perform as well as the Garmin. Only about $10 \%$ of the data points were real-time corrected, which was a poorer performance than expected. Previous use of the system for area surveys in the region has indicated that the beacon correction system might work well, and it had been hoped that it would provide a comparison of the correction by field structure and by DGPS. The data presented in this paper are not sufficient to make comment on the utility of the service in the centre of the country, but given that the service is aimed at marine users, it is not surprising that a problem occurred at the geographical centre of the country.
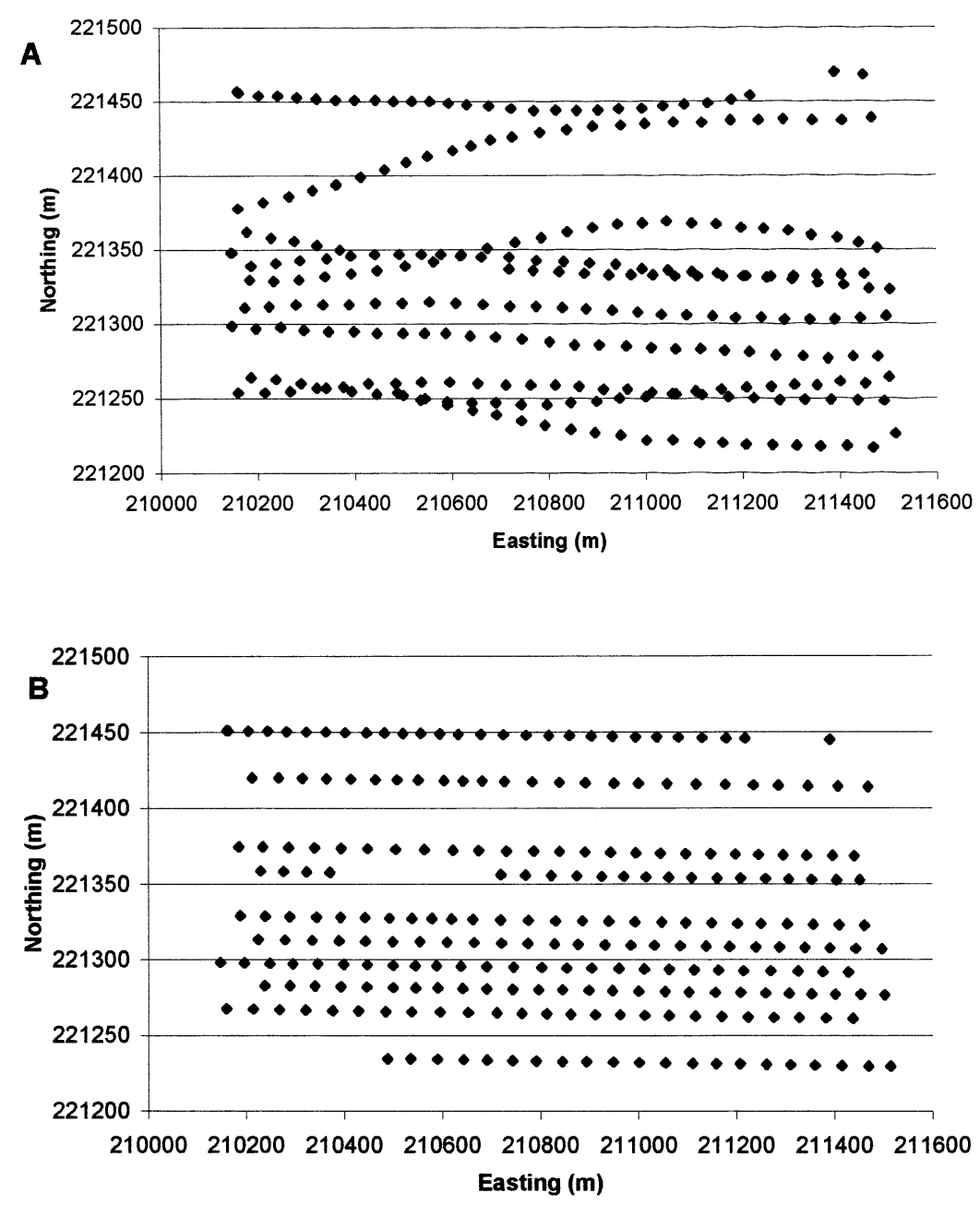

Fig. 4. A raw track data set (A) and corrected positions to field centrelines (B). 
Using the first data point after the turn (Garmin data), the correct field was consistently chosen $60 \%$ of the time. Of the misallocated fields half were adjacent (i.e. $15 \mathrm{~m}$ error) and half were not adjacent (30-60-m error).

\section{Discussion}

\subsection{Onfield or headland?}

The position with respect to a boundary line can be reliably determined even with non-corrected GPS data. It can be seen in Fig. 4A that the data error is smooth, probably due to the relatively coarse sampling interval (10 s) which had to be used for these tests to ensure that data memory capacity was not exceeded. There is, however, no reason to believe that there would be rapid changes in heading if the data density was greater. There were no outlying data points in the headland area, leading to field/headland designation being reliable. One possible reason for this is that, when the machine is moving, the position fixing algorithm may not permit a point to be recorded that is contrary to the direction of movement, and as a point is calculated every $10 \mathrm{~s}$, there is little chance of this happening due to the distance travelled between points. Managing the $\sim 50-\mathrm{m}$ error associated with over estimating the number of points on a turn can be achieved by analysis of forward speed. The time required for a particular turn at the current speed could be used to reduce error. It is the arrival at a turn that is the most important event to recognise and this can be done reliably.

\subsection{Position onfield}

The position along a field could be obtained by correction with an average error of $\sim 10 \mathrm{~m}$, which represented a significant improvement in accuracy over uncorrected position. Working at this general resolution, the pseudo-differential correction by field line would permit the use of non-DGPS equipment on the bog for industrial peat production. The specification of positioning accuracy required for miller control has not been reliably calculated but given that machine control will be based on discrete classes and not continuous adjustment, a resolution of 10 $\mathrm{m}$ is probably acceptable. In view of the rapidly advancing technology and reductions in hardware costs the approach suggested here may well be superseded by a more cost effective DGPS. However the method presented still provides an excellent means of calculating position under circumstances when either differential correction signals are not available (or are unreliable) or the added mechanical and financial costs of a DGPS are undesirable. It should also be noted, that a series of parallel lines are not required to implement the system, just straight lines, so it could also be applied to the bog railway system and other bog management operations. 


\subsection{Which field is the machine on?}

The underlying assumption behind the system as presented, is that it is always known which field the machine is on. If however this assumption cannot be met, we can see that the correct field was selected after a turn $60 \%$ of the time (consistently over the three data sets using the Garmin data). If we also consider that, of the misallocated fields, half resulted in a $15 \mathrm{~m}$ error, then we can be sure that error is constrained to $15 \mathrm{~m}$ for about $80 \%$ of the time. For industrial peat production this is probably reliable enough because current technology for generating application maps (i.e. Landsat Thematic Mapper imagery; McGovern et al., 1998) has a resolution of $30 \mathrm{~m}$. If the correction method were applied to a DGPS to improve the fix further, or for those occasions when differential signal could not be obtained, then knowledge of which field would be of less significance.

\subsection{Implications for precision peat production}

Ward and Holden (1998) proposed a precision peat production (PPP) system that would utilise precision agriculture technology to optimise the milling (and therefore drying) of milled peat based on four steps: (i) mapping milled peat density distribution from satellite imagery (McGovern et al., 1998); (ii) establishing ideal milling depth for each peat type on the bog using a weather forecast to predict 4-day drying conditions and a model of milled peat drying; (iii) a GPS equipped tractor hauling a milling machine controllable for depth with sinkage compensation; and (iv) an integrated decision support system to automate the management of the spatial information. On the basis of the evaluation of low-cost GPS performance and pseudo-differential correction it can be concluded that the method has scope to be relevant to application in the peat industry without DGPS being necessary. It would be necessary to implement a linked and automated method of field identification or to operate a milling procedure by consecutive fields. The fact that a low-cost GPS can be made accurate to $\sim 10 \mathrm{~m}$ indicates that even if such a constraint was not applied then the system could still work acceptably within the 30-m resolution of Landsat Thematic Mapper imagery. This imagery is highly suited to producing density maps provided that bog management was such that occasional 'high' fields were accounted for by manual over-ride. A full field width miller (currently a half-field width machine is used) would also be desirable for best performance with the GPS system.

\section{Conclusion}

In conclusion, it has been demonstrated that by use of imposed field structure, low-cost GPS can be used with an accuracy, on average, of about $10 \mathrm{~m}$, which is a significant improvement on the quoted $100 \mathrm{~m} 2 \mathrm{dRMS}$. The requirements for achieving such accuracy are fixed, straight lines of travel which have been accurately surveyed, and a knowledge of when one known point has been passed in order to establish the first correction calculation. 


\section{References}

Beuche, H., Hellebrand, H.J., 1998. Positionsgenauigkeit verfugbarkeit verbessern-fahrwegberechnung und lineare regressionsanalyse stutzen DGPS. Landtechnik 53, 140-141.

LaComber, P., Ward, S.M., Lynch, J., 1997. The effect of particle size on the drying of milled peat. Drying Technol. 15, 1083-1093.

McGovern, E.A., Holden, N.M., Ward, S.M., Collins, J.F., 1998. Calibration of Landsat Thematic Mapper imagery for milled peat density prediction. In: The Spirit of Peatlands. Jyvaskyla, Finland International Peat Society, Jyvaskyla, Finland, pp. 249-251.

von Ow, C., Ward, S.M., Lynch, J., 1996. Peat compressibility: a parameter for automatic depth control of milling machines. J. Agric. Eng. Res. 63, 295-300.

Ward, S.M., Holden, N.M., 1998. Precision peat production. In: Robert, P.C., Rust, R.H., Larsen, W.E. (Eds.), Proceedings of the 4th International Conference on Precision Agriculture. ASA/CSSA/SSSA, Madison, WI. 